Subject 1, Subsection 1.2 New Materials and Concepts for Cells

\title{
Quantum Wire-on-Well (WoW) Cell with Long Carrier Lifetime for Efficient Carrier Transport
}

\author{
Hiromasa Fujii ${ }^{1 *}$, Takumi Kato ${ }^{1}$, Kasidit Toprasertpong ${ }^{1}$, Hassanet Sodabanlu ${ }^{2}$, Kentaroh Watanabe ${ }^{2}$, Diego \\ Alonso-Álvarez ${ }^{3}$, Megumi Yoshida ${ }^{3}$, Nicholas Hylton ${ }^{3}$, Nicholas Ekins-Daukes ${ }^{3}$, Masakazu Sugiyama ${ }^{1}$, and \\ Yoshiaki Nakano ${ }^{1}$ \\ 1 Department of Electrical Engineering and Information Systems, the University of Tokyo \\ 2 Research Center for Advanced Science and Technology, the University of Tokyo \\ 3. Department of Physics, Imperial Collage, London \\ *Address: \#230, Eng. Bldg. 10, the Univ. of Tokyo, Yayoi, Bunkyo-ku, Tokyo, 113-0032, Japan \\ Phone: +81-3-5841-6753, Fax: +81-3-5841-6027, E-mail: fujii@hotaka.t.u-tokyo.ac.jp
}

\begin{abstract}
SUMMARY: A quantum wire-on-well (WoW) structure, taking advantage of the layer undulation of an InGaAs/GaAsP superlattice (SL) grown on a vicinal substrate, was proved to enhance the carrier collection from the confinement levels as compared with a planar reference SL, and the carrier lifetime in the WoW was approximately 4 times longer than the planar SL. SLs grown on vicinal substrates are likely to be undulated due to formation of step bunching [1]. It was suggested that the InGaAs wire structure in an undulated InGaAs/GaAsP SL extends carrier lifetime [2]. This study provides a fare comparison between undulated and planar InGaAs/GaAsP SL, which were grown under exactly the same condition except for the substrate misorientation $\left(0^{\circ}\right.$ - and $6^{\circ}$ - off $)$, and the advantage of the undulated SL was confirmed. In our InGaAs/GaAsP SL, the step bunching occurred especially in InGaAs layers, and periodically aligned InGaAs nanowires emerged on planar wells. Light absorption for such a structure was degraded due to poorer in-plane coverage of InGaAs, but the collection of the photo-excited carriers was more efficient than for a planar SL, possibly because of tunnel transport through locally thinned GaAsP barriers. Another possible mechanism for the enhanced carrier collection is an extremely-long carrier lifetime $(220 \mathrm{~ns})$, which will be beneficial not only for the bandgap engineering using quantum structures but also for the realization of intermediate-band cells.
\end{abstract}

GaAs pin cells including 50-period $\mathrm{In}_{0.30} \mathrm{Ga}_{0.70} \mathrm{As}(3.5 \mathrm{~nm}) / \mathrm{GaAs}(2.7 \mathrm{~nm}) / \mathrm{GaAs}_{0.60} \mathrm{P}_{0.40}(3.0 \mathrm{~nm}) / \mathrm{GaAs}(2.7$ $\mathrm{nm}) \mathrm{SLs}$ in the i-regions were prepared on GaAs $\left(\begin{array}{lll}0 & 0 & 1\end{array}\right)$ substrates. To investigate the effect of layer undulation, one cell was grown on an exact-oriented substrate, and the other was grown on a vicinal substrate $6^{\circ}$ misoriented toward [ $\left[\begin{array}{lll}1 & 1 & 1\end{array}\right]$ direction (Fig. 1). STEM observation revealed that the SL grown on the vicinal substrate was significantly undulated due to step bunching, forming InGaAs wires on wells (WoW), whereas the SL grown on the exact-oriented substrate was quite planar (Fig. 2 (a)-(b)). The lateral periodicity of the layer undulation was approximately $67 \mathrm{~nm}$, which was also confirmed by 2-dimensional fringe pattern of XRD-RSM (Fig. 2(c)-(d)).

Incorporation of SLs extended the absorption edge to longer wavelength owing to the narrow-gap InGaAs regions (Fig. 3). The planar SL on the exact-oriented substrate showed typical step-like absorbance spectra with a sharp exciton peak at $1005 \mathrm{~nm}$. The WoW on the vicinal substrate, on the other hand, exhibited a monotonically declining absorption tail with red-shifted absorption edge, because of lateral variation of quantum confinement in the thickness-modulated wells. The step-bunching in InGaAs also lowered the in-plane coverage of the well region, resulting in significant reduction of the overall absorbance.

The carrier transport was enhanced in the WoW compared to the uniform SL. Carrier collection efficiency (CCE) under AM1.5G illumination was preserved over $99 \%$ up to $0.65 \mathrm{~V}$ for the WoW, whereas it apparently decreased with forward bias for the planar SL (Fig. 4). The short circuit current, therefore was comparable between the two cells in spite of the reduced light absorption in the WoW. Consequently, the WoW cell on a vicinal substrate showed higher efficiency than the SL cell on an exact-oriented substrate, in particular owing to enhanced FF. (Fig. 5, Table 1). In the SL, the carriers in the InGaAs wells are thermally excited to the upper states and then tunnel through the thin GaAsP barriers [3]. The enhanced carrier transport in the WoW, therefore, would be attributed to enhanced tunnel transport through locally thin GaAsP area formed due to the thickness modulation.

To examine the carrier lifetime in both the WoW and the planar SL, only the SL regions with the same structures were grown on p-type GaAs substrates for PL measurement (Fig. 6). The WoW showed a broader PL spectrum with luminescence at longer wavelength due to the locally weakened quantum confinement. The PL decay time constant after $4.8 \mathrm{pJ}$ pulse excitation at $780 \mathrm{~nm}$ was $50 \mathrm{~ns}$ and $220 \mathrm{~ns}$ for the planar SL and the WoW, respectively, which might be another reason for higher CCE of the WoW under operational forward bias.

\section{References}

[1] N.Y. Jin-Phillipp et al., J. Mater. Sci: Mater Electron, 8 (1997) 289-299.

[2] D. Alonso-Álvarez, et al., Appl. Phys. Lett. 105 (2014) 083124.

[3] H. Fujii et al., Prog. Photovolt: Res. Appl. 22 (2013) 784-795. 


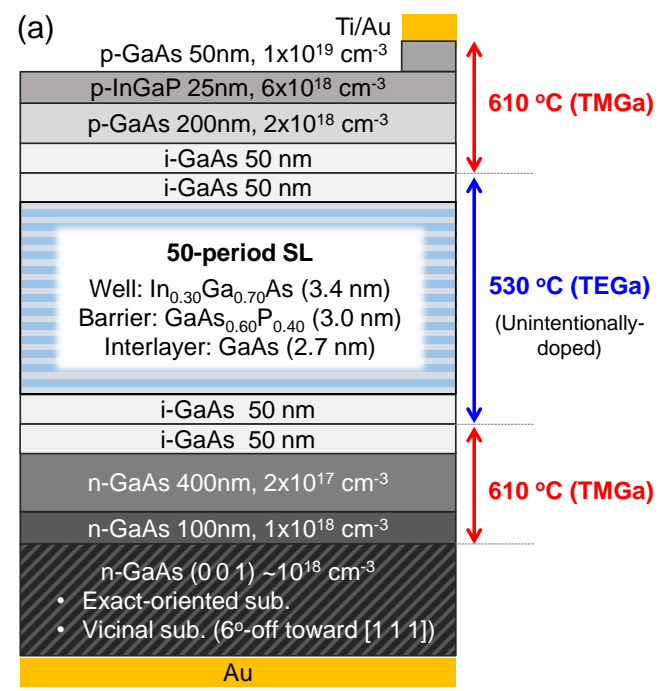

(b)

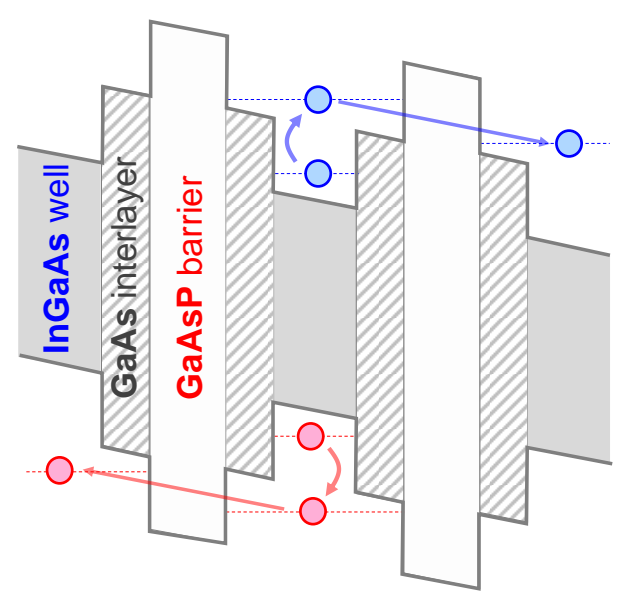

Fig. 1 (a) GaAs pin cells including 50-period InGaAs/GaAsP superlattice (SL) fabricated in this study. Either an exact-oriented $\left(\begin{array}{lll}0 & 0 & 1\end{array}\right)$ substrate or a misoriented substrate $6^{\circ}$ cutoff toward [ $\left.\begin{array}{lll}1 & 1 & 1\end{array}\right]$ were used for the planar SL and the well on wire (WoW), respectively. The p- and n-region were grown at $610^{\circ} \mathrm{C}$ using trimethylgallium (TMGa) and the SLs were grown at $530^{\circ} \mathrm{C}$ using tryehylgallium (TEGa). The entire SLs were compressively strained at $2560 \mathrm{ppm}$ due to intentionally thinned GaAsP barriers for efficient tunnel transport. (b) Schematic of carrier transport process in the designed SL; the photo-excited carriers in the InGaAs wells can tunnel through the GaAsP barriers after being thermally excited to the upper states.

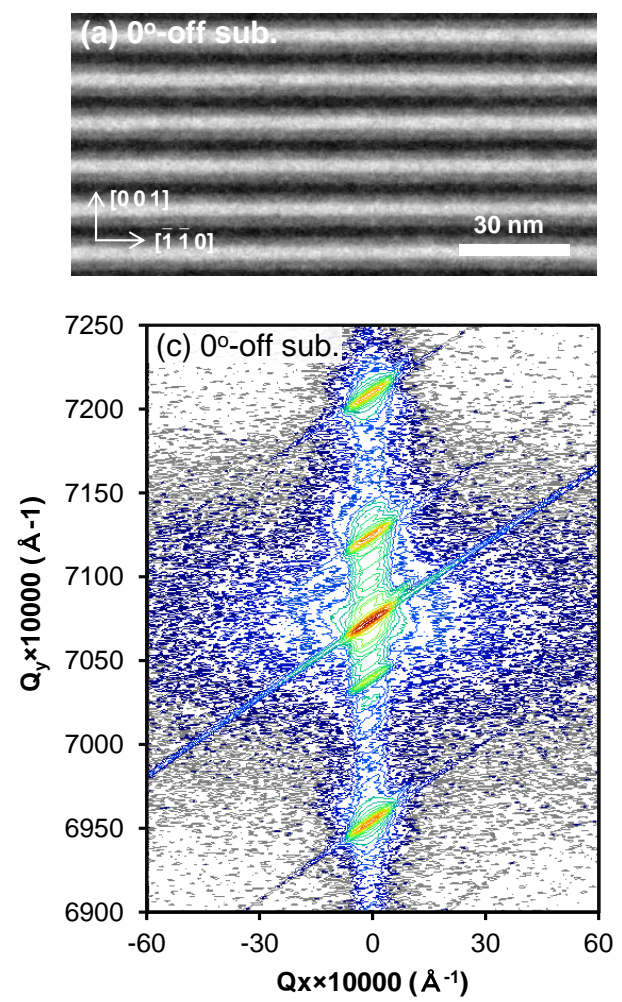

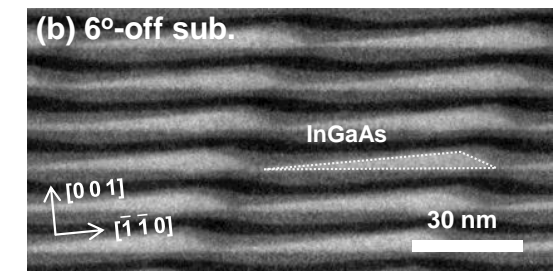

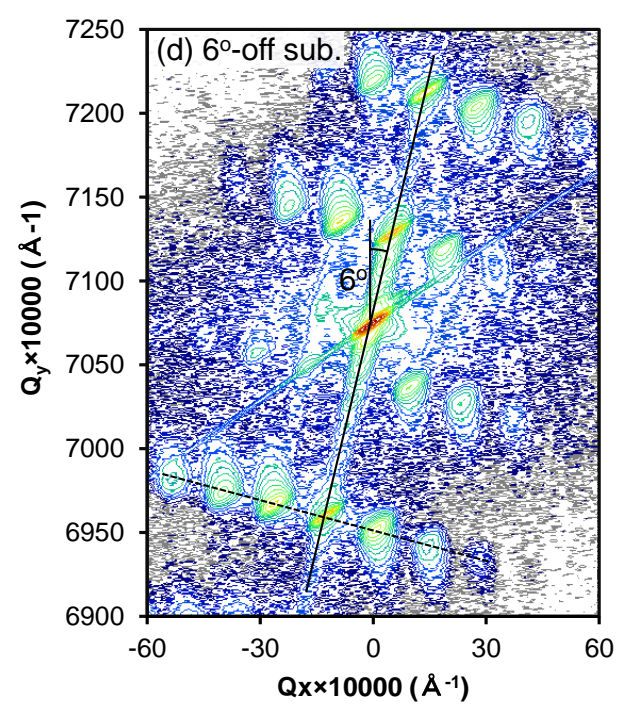

Fig. 2 Magnified HAADF STEM images of (a) the planar SL grown on an exact-oriented, and (b) the WoW on a vicinal substrate. The grown SL was very uniform on the exact-oriented substrate whereas it was significantly undulated on the vicinal substrate due to step bunching, which especially occurred in InGaAs region with extended $\left(\begin{array}{lll}0 & 0 & 1\end{array}\right)$ terrace. (c) (d) XRD-RSM around the GaAs ( $\left.\begin{array}{llll}0 & 0 & 4\end{array}\right)$ diffractions points. The planar SL grown on the exactoriented substrate showed one-dimensional fringe pattern along the $\mathrm{Q}_{\mathrm{y}}$-axis, which represents the periodicity in the growth direction. The WoW on a vicinal substrate showed 2-dimensional fringe pattern due to laterally periodic layer undulation, for which the periodicity can be estimated from the peak interval along the dotted line in (d). 


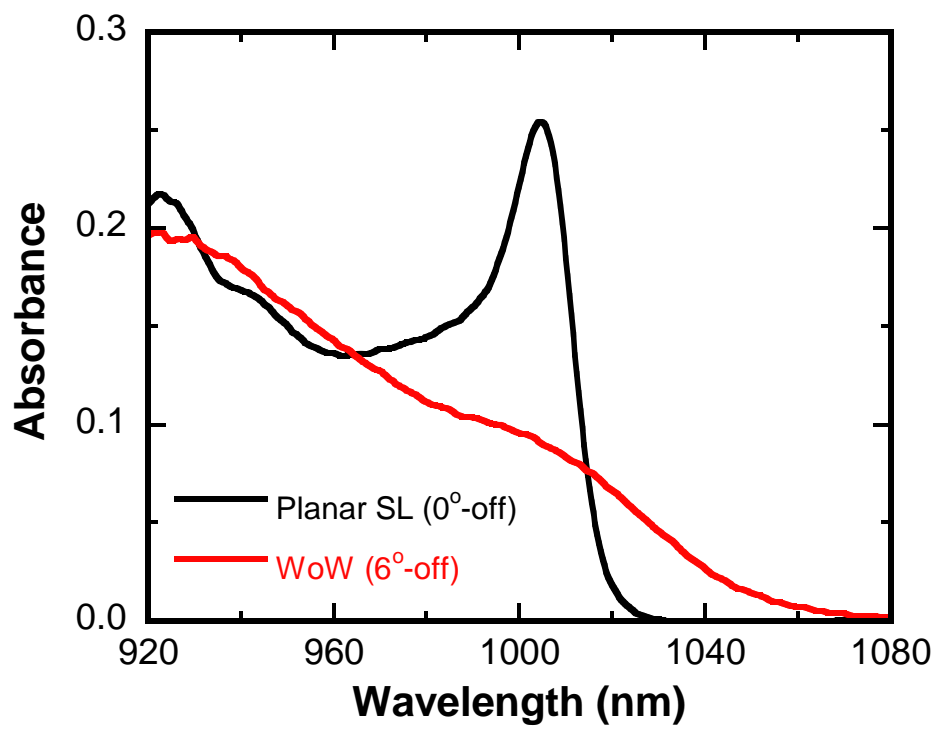

Fig. 3 Absorbance spectra for the 50-period SLs grown on an exact-oriented ( $0^{\circ}$-off) substrate or a vicinal $\left(6^{\circ}\right.$ off) substrate. The planar SL on a $0^{\circ}$-off substrate showed a typical step-like spectrum with a sharp exciton peak at $1005 \mathrm{~nm}$. The WoW on a $6^{\circ}$-off substrate showed a gradually declining absorption tail with a red-shifted spectrum edge due to lateral variation of the quantum confinement level. This contributed to additional absorption in 1020-1070 nm, though the absorption in 970-1010 nm was significantly lowered because of the poorer in-planecoverage of InGaAs; the areas between the wires in the WoW are covered by extremely thin InGaAs where longwavelength light cannot be absorbed.

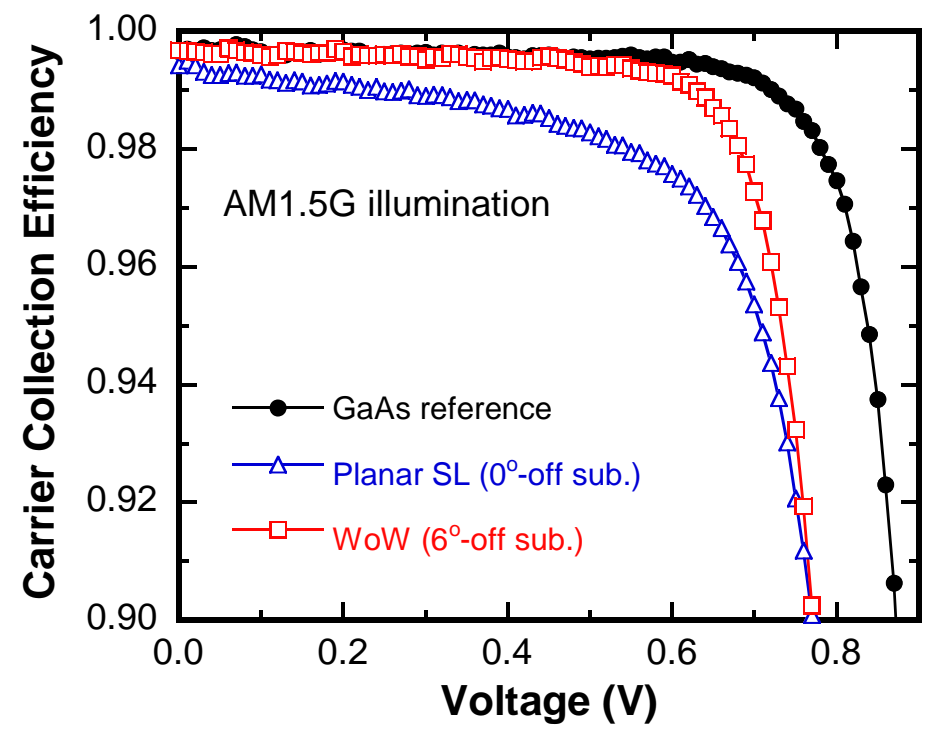

Fig. 4 Carrier collection efficiency (CCE) under AM1.5G illumination. CCE was estimated by normalizing the illumination-induced current enhancement to the saturation value at reverse bias. A result for a GaAs reference cell is shown together. CCE showed apparent declination for the cell with the planar SL on an exact-oriented $\left(0^{\circ}\right.$ off) substrate, whereas it was preserved over $99 \%$ up to $0.6 \mathrm{~V}$ for the cell with the WoW on a vicinal $\left(6^{\circ}\right.$-off) substrate, resulting in CCE at maximum power output voltages of $95.4 \%$ and $96.8 \%$, respectively. The enhanced carrier transport in the WoW should be attributed to efficient tunneling through the thin GaAsP region, and/or the long carrier lifetime as revealed by TRPL measurement (Fig. 7(b)). 

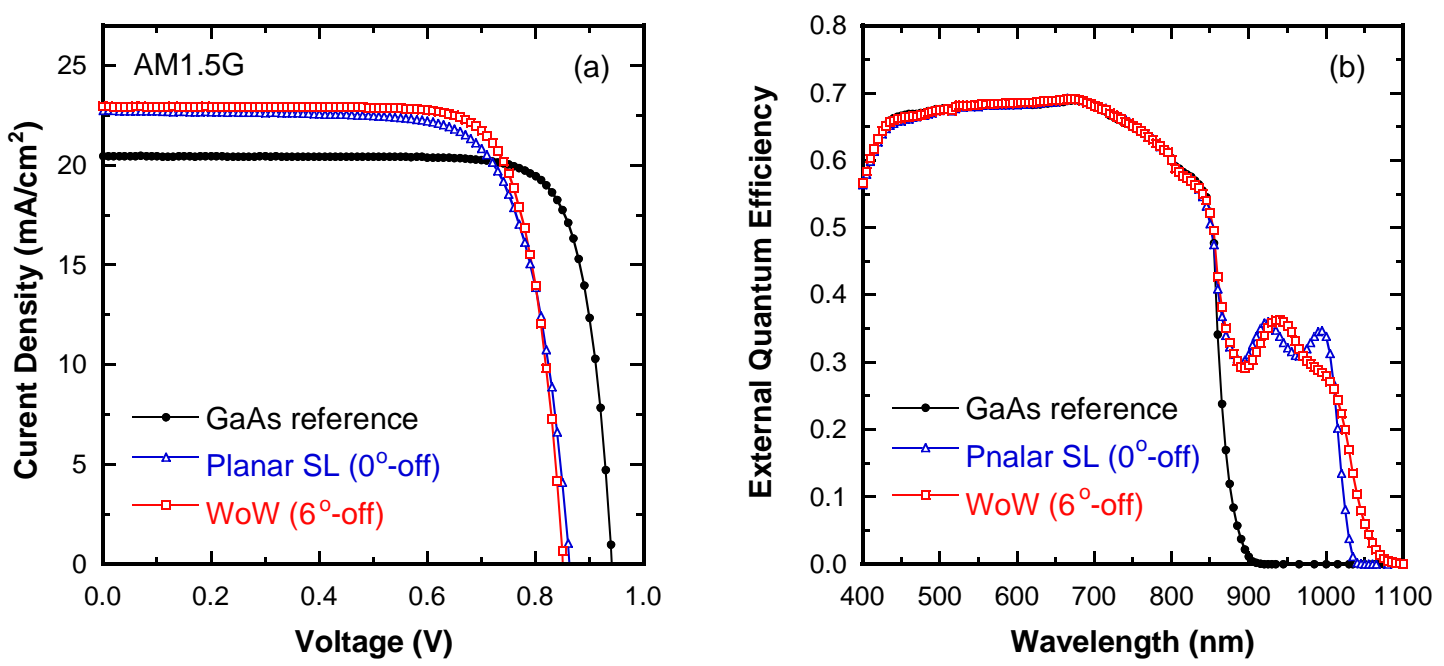

Fig. 5 (a) I-V characteristics under AM1.5G and (b) external quantum efficiency at $0 \mathrm{~V}$ for the cells with the 50period planar SL ( $0^{\circ}$-off) and the 50-period WoW ( $6^{\circ}$-off) and the GaAs reference cell. Anti-reflection coating was not applied to the devices in this work.

Table 1 Cell performance of the fabricated PV devices under AM1.5G (full spectrum), and AM1.5G filtered by a 665-nm long-pass filter (FGL665S, THORLABS). The filtered illumination was used for evaluation of the performance as a second sub-cell beneath an InGaP top cell in a multi-junction device. Note that the efficiency under the filtered AM1.5G was calculated with the filtered incident illumination energy of $514 \mathrm{~W} / \mathrm{m}^{2}$.

\begin{tabular}{l|cccc|cccc}
\hline & \multicolumn{4}{|c|}{ AM1.5G (full spectrum) } & \multicolumn{4}{c}{ AM1.5G filtered (> 665 nm) } \\
\cline { 2 - 9 } & $\mathrm{J}_{\mathrm{sc}}\left(\mathrm{mA} / \mathrm{cm}^{2}\right)$ & $\mathrm{V}_{\mathrm{oc}}(\mathrm{V})$ & $\mathrm{FF}$ & Efficiency & $\mathrm{J}_{\mathrm{sc}}\left(\mathrm{mA} / \mathrm{cm}^{2}\right)$ & $\mathrm{V}_{\mathrm{oc}}(\mathrm{V})$ & FF & Efficiency \\
\hline GaAs ref & 20.5 & 0.942 & 0.810 & $15.6 \%$ & 8.17 & 0.915 & 0.802 & $11.6 \%$ \\
\hline Planar SL (0-off sub) & 22.8 & 0.863 & 0.743 & $14.6 \%$ & 10.4 & 0.825 & 0.749 & $12.5 \%$ \\
\hline WoW (6 ${ }^{\circ}$-off sub.) & 23.0 & 0.852 & 0.780 & $15.3 \%$ & 10.4 & 0.822 & 0.783 & $13.0 \%$ \\
\hline
\end{tabular}
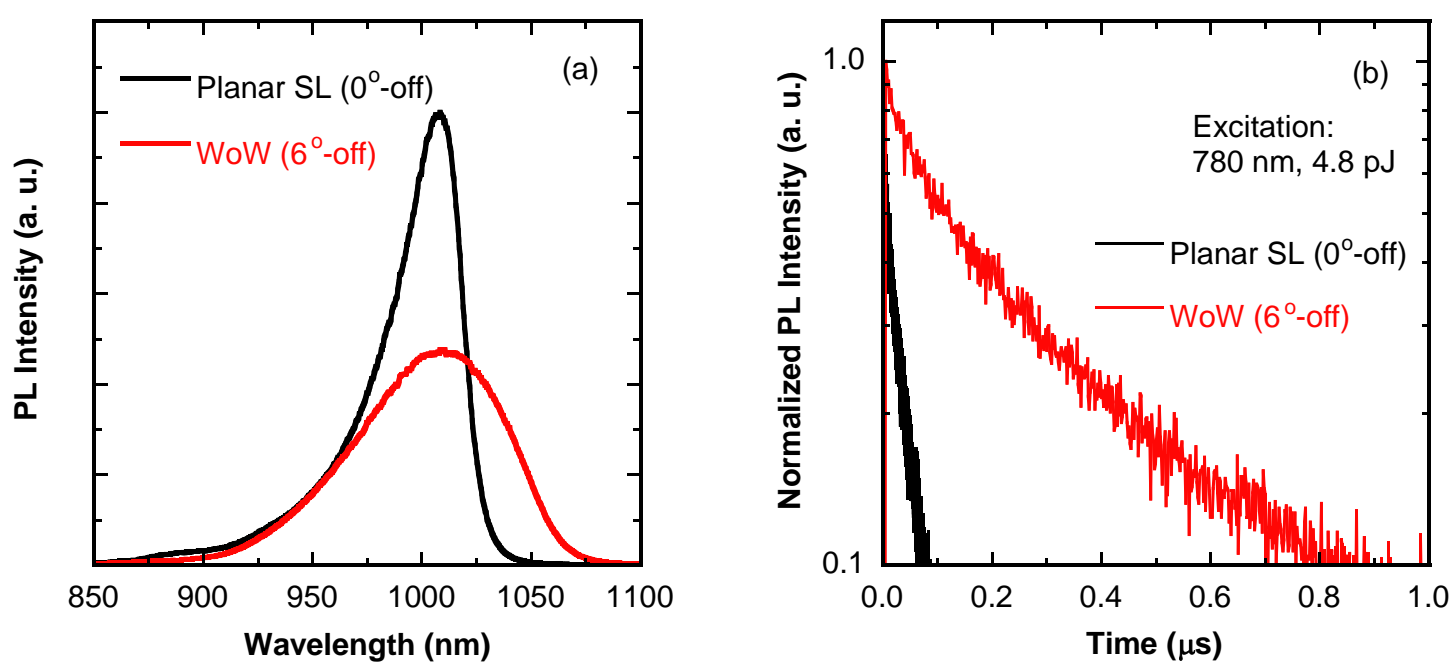

Fig. 6 (a) PL spectra for the planar SL grown on an exact-oriented ( $0^{\circ}$-off) substrate and the WoW on a vicinal $\left(6^{\circ}\right.$-off) substrate at excitation wavelength of $780 \mathrm{~nm}$. The samples consisted of only unintentionally-doped SLs with p-type background doping grown on p-type substrate to ensure flat band-lineup for the quantum structures. The WoW on a vicinal substrate showed broader peak with luminescence at longer wavelength due to the locally weakened quantum confinement. (b) PL decay curves at the peak wavelength measured for the same samples. The estimated carrier lifetimes were $50 \mathrm{~ns}$ and $220 \mathrm{~ns}$ for the planar SL and the WoW, respectively. 